\title{
Klotho, an anti-aging gene, acts as a tumor suppressor and inhibitor of IGF-1R signaling in diffuse large B cell lymphoma
}

Xiangxiang Zhou' ${ }^{1}$ Xiaosheng Fang ${ }^{1}$, Yujie Jiang ${ }^{1}$, Lingyun Geng ${ }^{1}$, Xinyu Li ${ }^{1}$, Ying Li ${ }^{1}$, Kang Lu' ${ }^{1}$ Peipei Li ${ }^{1}$, Xiao Lv $^{1}$ and Xin Wang ${ }^{1,2^{*}}$

\begin{abstract}
Background: Klotho, is a transmembrane protein, performs as a circulating hormone and upstream modulator of the insulin-like growth factor-1 receptor (IGF-1R), fibroblast growth factor (FGF), and Wnt signaling pathways. These pathways are involved in the development and progression of B cell lymphoma. We aimed to explore the expression pattern and functional mechanism of Klotho in diffuse large B cell lymphoma (DLBCL).

Methods: Immunohistochemistry $(\mathrm{IHC})$ and western blotting were performed to detect the expression level of Klotho in DLBCL patients and cell lines. Tumor suppressive effect of Klotho was determined by both in vitro and in vivo studies. Signaling pathway activity was assessed by western blotting.

Results: Remarkable lower expression levels of Klotho were observed in DLBCL patients and cell lines. Enforced expression of Klotho could significantly induce cell apoptosis and inhibit tumor growth in DLBCL. Upregulation of Klotho resulted in declined activation of IGF-1R signaling, accompanied with decreased phosphorylation of its downstream targets, including AKT and ERK1/2. Moreover, xenograft model treated with either Klotho overexpression vector or recombinant human Klotho administration presented restrained tumor growth and lower Ki67 staining.
\end{abstract}

Conclusions: Our findings establish that Klotho performs as a tumor suppressor and modulator of IGF-1R signaling in DLBCL. Targeting Klotho may provide novel strategies for future therapeutic intervention.

Keywords: Diffuse large B cell lymphoma, Klotho, Insulin growth factor-1 receptor, Tumor suppressor

\section{Background}

Diffuse large B cell lymphoma (DLBCL) is the most common form of non-Hodgkin lymphoma (NHL), accounts for nearly $40 \%$ of all newly diagnosed cases [1]. This disease presents as an aggressive process and exhibits high heterogeneity in gene expression and clinical outcomes [2, 3]. Although majority of DLBCL patients could be cured by anthracyclinebased chemotherapies combined with rituximab, one third of them presented refractory or relapsed process $[4,5]$. Therefore, more effective treatment strategies

\footnotetext{
*Correspondence: xinw@sdu.edu.cn; xinw007@126.com

'Department of Hematology, Shandong Provincial Hospital affiliated to

Shandong University, No.324, Jingwu Road, Jinan, Shandong 250021,

People's Republic of China

${ }^{2}$ Shandong University School of Medicine, Jinan, Shandong 250012, People's Republic of China
}

based on novel therapeutic targets and molecular cogenic pathways are still needed. Klotho is an anti-aging gene originally identified in
1997 [6]. Kuro-o et al. [6, 7] found that Klotho-deficient of mice. The Klotho gene is located in chromosome $13 \mathrm{q} 12$ in human with the size of $50 \mathrm{~kb}$ [6]. It encodes a single-pass transmembrane protein, which consists of an extracellular domain, a single transmembrane domain, and an intracellular domain. The intracellular domain is very short and has no clear functions. Membrane Klotho functions as an obligate co-receptor of fibroblast growth factor 23 (FGF23) to regulate phosphate homeostasis [8]. The extracellular domain (secreted Klotho) could be released into the serum and functions as a circulating mice developed multiple premature aging syndromes, 
hormone to regulate the activity of oxidative stress, multiple growth factor receptors, and ion channels $[9,10]$.

The tumor suppressive activity of Klotho was first identified in breast cancer in 2008 [11]. Recent investigations have implicated that Klotho is extensively downregulated in several solid tumors, including cervical cancer, pancreatic cancer, melanoma, and several digestive neoplasm [12]. In these malignancies, Klotho was elucidated to be a modulator of several signaling pathways, including the FGF signaling, insulin-like growth factor-1 receptor (IGF-1R), and Wnt pathways, which are also involved in the pathogenesis of hematological malignancies [10, 13-15]. However, the role of Klotho in hematological malignancies has not been reported.

A large number of aberrant receptor tyrosine kinases (RTKs) have been found in hematological malignancies $[16,17]$, but they are still indefinite in DLBCL. IGF-1R is a RTK primarily activated by its cognate ligands, IGF-1, and IGF-2. It plays a crucial role in the establishment and progression of tumors by regulating proliferation, self-renewal, apoptosis, and drug resistance of cancer cells [18-20]. Activation of IGF-1R following IGF-1 treatment results in phosphorylation of downstream signaling cascades, including PI3K/AKT and MAPK/ERK [19]. Blockade of PI3K/AKT signaling could restrain cell survival and function of lymphocytes [21, 22]. Activation of MAPK/ERK signaling promotes cell proliferation and metastasis of cancer cells [23]. Strategies to block IGF-1R pathway in solid malignancies are being tested in clinical trials [24], whereas the function of IGF-1R signaling in DLBCL has been less studied [25].

In this present study, we aimed to assess the expression level and functional mechanism of Klotho in DLBCL. We identified reduced expression of Klotho in DLBCL for the first time. Noted inhibition of cell growth and induction of apoptosis were observed in DLBCL with Klotho overexpression. Tumor growth was restrained by administration of Klotho protein in xenograft model of DLBCL. Our findings demonstrated that Klotho was a tumor suppressor and modulator of IGF-1R signaling in DLBCL, indicating that targeting Klotho may provide novel therapeutic strategy in DLBCL.

\section{Methods}

\section{Patients}

This study was approved by the Medical Ethical Committee of Shandong Provincial Hospital affiliated to Shandong University. The paraffin-embedded archived samples from 50 newly diagnosed DLBCL patients and 20 reactive hyperplasia patients were collected. Samples of patients with reactive hyperplasia were referred as control. Histological diagnoses were established according to the WHO classification [26]. Peripheral blood mononuclear cells (PBMCs) from the whole blood of healthy donors were isolated using Ficoll-Hypaque density gradient centrifugation (TBD science, Tianjin, China). Normal peripheral blood CD19+ B cells were purified from freshly isolated PBMCs using CD19+ magnetic microbeads kit (Miltenyi Biotec, Bergisch Gladbach, Germany). Cells were incubated with beads for $15 \mathrm{~min}$ at $4{ }^{\circ} \mathrm{C}$ while rotating. Purified $\mathrm{CD} 19+\mathrm{B}$ cells were selected according to the manufacturer's protocols. The purity of isolated populations was assessed by FACS analysis, and cells with $>90 \%$ purity were collected. All samples were obtained with informed consent in accordance with the Declaration of Helsinki.

\section{Cell lines and reagents}

Human DLBCL cell lines LY1 and LY8 were cultured in Iscove modified Dulbecco medium with $10 \%$ heatinactivated fetal bovine serum. The medium contains $1 \%$ penicillin/streptomycin mixture and $2 \mathrm{mmol} / \mathrm{l}$ glutamine. CD19+ B cells and PBMCs obtained from three healthy donors were used as controls $(\mathrm{N} 1, \mathrm{~N} 2$, and N3 cells). Recombinant human Klotho (rhKL) and recombinant human IGF-1 were obtained from PeproTech (Rocky Hill, NJ, USA), and adriamycin (ADR) was bought from Actavis (S.p.A, Italy).

\section{Cell transfection}

Lentivirus vectors either encoding Klotho or an empty lentiviral vector were from Genechem (Shanghai, China). Lentivirus transfection was carried out according to the manufacturers' instruction. Infection efficiencies were assessed by green fluorescent protein (GFP) through flow cytometry. The stably transfected cells were selected $48 \mathrm{~h}$ later with $5 \mu \mathrm{g} / \mathrm{ml}$ puromycin (Sigma-Aldrich, USA).

\section{Quantitative real-time PCR}

Total RNA was extracted using RNAiso Plus (TaKaRa, Dalian, China). Reverse transcription reaction was conducted with the reverse transcription reagents (TaKaRa, Dalian, China). Amplification reactions were performed with SyberGreen (TaKaRa, Dalian, China) in LightCycler 480II (Roche, Basel, Swizerland). Klotho-specific primers were as follows: forward, $5^{\prime}$-AGCAATCTGGTCTGAAT AACACTGG; reverse, $5^{\prime}$-CATGTTTCAGCGTGAAAG TTCAAAG. Relative quantification was calculated using the ${ }^{\Delta \Delta} \mathrm{CT}$ method.

\section{Immunohistochemistry (IHC) and hematoxylin-eosin staining}

The 4- $\mu$ m-thick formalin-fixed, paraffin-embedded tissue sections were deparaffinized and hydrate. Antigen retrieval was performed using $0.01 \mathrm{~mol} / \mathrm{l}$ sodium citrate buffer ( $\mathrm{pH}$ 6.0) under high pressure followed by a 1-h cool-down and rinses in phosphate buffer solution 
(PBS). Endogenous peroxidase was blocked with 3\% hydrogen peroxide in methanol for $15 \mathrm{~min}$, followed by incubation with normal serum to block non-specific binding. The slides were then incubated overnight at $4^{\circ}$ C with primary antibodies, anti-Klotho (1:150) or antiKi67 (1:100). After washing, the tissue sections were treated with the second antibody from SP reagent kit (Zhongshan Goldenbridge, Beijing, China) for $30 \mathrm{~min}$ at room temperature, followed by further treated with strept avidin-horseradish peroxidase complex (SABC) for $30 \mathrm{~min}$ at room temperature. After treated with diaminobenzidine (DAB) Kit (ZhongshanGoldenbridge, Beijing, China), the stained slides were counterstained with hematoxylin and mounted. Negative control was carried out with the primary antibody replaced by PBS. IHC staining was scored by the proportion of positive tumor cells. Five microscopic fields with the highest immunoreactivity at $\times 400$ magnification were evaluated by two independent observers who were blinded to the patients' clinical data. Cases with at least $10 \%$ of tumor cells with Klotho staining were considered as positive. Fresh mice subcutaneous tumors were fixed in $4 \%$ paraformaldehyde (PFA) and embedded with paraffin for histological examinations. Sections with $4-\mu \mathrm{m}$ thickness were cut and stained with hematoxylin-eosin (H\&E).

\section{Western blotting}

Cells were lysed in radio-immunoprecipitation assay buffer (Shenergy Biocolor, Shanghai, China) together with $1 \times$ phosphatase inhibitor cocktail (PhosSTOP; Roche, Mannheim, Germany). The BCA assay (Shenergy Biocolor, Shanghai, China) was performed to detect protein concentration. Protein extracts $(30 \mu \mathrm{g})$ were then electrophoresed on SDS-polyacrylamide gels and blotted from the gel onto polyvinylidene fluoride membranes (Millipore, Billerica, MA, USA). Membranes were incubated with the blocking solution (5\% skim milk in Tris-buffered saline containing $0.05 \%$ Tween-20) for $1 \mathrm{~h}$ at room temperature and then immunoblotted with the indicated antibodies (1:1000 dilution) overnight at $4^{\circ}$ C. After which, the membranes were washed with TBS-T and then probed with the HRP-conjugated secondary antibodies (Zhongshan Goldenbridge, Beijing, China) and the electro-chemi-luminescence kit (Millipore, Billerica, MA, USA). Chemiluminescent signals were detected using the Amersham Imager 600 imaging system (General Electric, USA). ImageJ software (ImageJ, NIH) was used to quantify the protein bands normalized to control. Primary antibodies used were Klotho (Abcam, Cambridge, MA, USA), phospho-IGF-1R (Tyr1135/1136), IGF-1R, phospho-AKT(Ser473), total pan-AKT, Mcl-1, Caspase-3, diphosphorylated and total ERK1/2 (Cell Signaling Technologies, Beverly, MA, USA), $\beta$-actin, and GAPDH (Zhongshan Goldenbridge, Beijing, China). The experiments were performed in triplicate with GAPDH or $\beta$-actin (Zhongshan Goldenbridge, Beijing, China) as endogenous control.

\section{Cell proliferation assay}

Cell proliferation was assessed by performing triplicate assays with the Cell Counting Kit-8 (CCK-8) assay (Enogene, Nanjing, China). DLBCL cells with designed treatment were seeded in 96-well plates at a density of 5000 cells/well for 48 or $24 \sim 96$ h later. Thereafter, the cells were incubated with $10 \mu \mathrm{l} /$ well CCK- 8 for $4 \mathrm{~h}$ according to the manufacturer's proposal. Cell proliferation was detected by light absorption at $450 \mathrm{~nm}$ by Multiskan GO Microplate Reader (Thermo Scientific, Rockford, IL, USA).

\section{Flow cytometry analysis}

Apoptosis of transfected DLBCL cells were detected by Annexin V-PE/7-aminoactinomycin (7AAD) (BD Biosciences, Bedford, MA, USA) assay according to the manufacturers' instructions. DLBCL cells with designed treatments were harvested and washed twice with icecold PBS and incubated in $1 \times$ binding buffer (containing $5 \mu \mathrm{l}$ Annexin V-PE and $5 \mu \mathrm{l}$ 7AAD). After incubation in the dark for $15 \mathrm{~min}$, cells were subjected to the flow cytometry. At least 10,000 events per sample were acquired. Cells were discriminated into viable cells (AnnexinV-PE ${ }^{-} / 7 \mathrm{AAD}^{-}$), dead cells (Annexin V-PE $-/ 7 \mathrm{AAD}^{+}$), apoptotic cells (Annexin $\left.\mathrm{V}-\mathrm{PE}^{+} / 7 \mathrm{AAD}^{-}\right)$, and necrotic cells (Annexin $\mathrm{V}-\mathrm{PE}^{+} / 7 \mathrm{AAD}^{+}$). The rates of apoptotic cells were acquired on a FACS-Navios Flow Cytometer (Beckman Coulter, CA, USA). Data were analyzed using FlowJo Version 7.6 software (Tree Star Inc., OR, USA).

\section{Elisa assay}

Serum soluble Klotho levels were measured using the Elisa kit (Immuno-Biological Laboratories, Gunma, Japan), with a lower limit of assay of $6.15 \mathrm{pg} / \mathrm{ml}$.

\section{In vivo xenograft study}

All animal experiments were performed in accordance with the principles of the Institutional Animal Care. Severe combined immunodeficiency (SCID) Beige female mice of 5-week old were bought (Weitong Lihua Laboratory Animal Center, Beijing, China) and maintained in a pathogen-free environment under controlled condition of light and humidity. $1 \times 10^{7}$ LY1 cells (untransfected, stably Klotho-overexpressing vector tranfected, or empty control vector transfected, respectively), mixed with $100 \mu \mathrm{l}$ Matrigel (BD Biosciences, Bedford, MA, USA), were subcutaneously injected into the right inferior limb of mice. Tumor size was measured by the digital caliper. For the experiment with rhKL, SCID Beige mice were 
injected subcutaneously with $1 \times 10^{7}$ LY1 cells into the left inferior limbs. Mice were treated with daily intraperitoneal injections of rhKL $(7.5 \mu \mathrm{g} / \mathrm{kg}$ ) or PBS control (six mice per group) for 2 weeks. The volume of tumor was estimated using the equation $V=\left(a \times b^{2}\right) \times 0.5236$, where $a$ is the largest dimension and $b$ is the perpendicular diameter.

\section{Statistical analysis}

All statistical analyses were performed by using the statistic software SPSS17.0 (SPSS Inc., Chicago, IL, USA) for Windows. In vitro experimental results were presented as mean \pm SD of data obtained from three separate experiments. Overall survival time was measured from the date of diagnosis to the date of death or the last follow-up. KaplanMeier analysis was performed to estimate the survival functions. A log-rank test was used to assess survival differences. Chi-square test was used to analyze the clinical characteristics of patients. One-way analysis of variance (ANOVA) or $t$ tests were used to assess the differences between groups. $p<0.05$ was considered to be statistically significant.

\section{Results}

Klotho was downregulated in DLBCL and related to tumor progression

Compared with reactive hyperplasia, expression level of Klotho was significantly lower in DLBCL tissues (Fig. 1a).
Klotho positive rate was $14 \%$ (7 of 50 ) in DLBCL whereas $80 \%$ (16 of 20 ) in reactive hyperplasia. To evaluate the clinical significance of Klotho in DLBCL, clinical and pathological characteristics of DLBCL patients were analyzed. The expression level of Klotho was associated with the Ann Arbor stage of DLBCL patients. Patients with stage III or IV presented markedly lower Klotho level than those with stage I or II $(p=0.002$, Table 1$)$. In addition, Kaplan-Meier survival analysis were performed based on the expression levels of Klotho $(n=50)$. The median survival of patients in Klotho-positive group was 48.46 months, significantly longer than those in Klothonegative group (29.27 months, $p=0.045$, Fig. $1 b)$. We next confirmed the expression of Klotho in DLBCL cell lines. Klotho mRNA expression was detected in DLBCL cell lines and normal CD19+ B cells. Significantly decreased levels of Klotho mRNA expression were observed in DLBCL cell lines (LY1 $0.024 \pm 0.037$, LY8 $0.002 \pm 0001$, Fig. 1c). Reduced expression levels of Klotho protein were also noted in DLBCL cell lines compared to human PBMCs (Fig. 1d).

\section{Klotho inhibited growth of DLBCL}

To further establish the biological function of Klotho, human DLBCL cells were stably transfected with either negative control lentivirus vectors (LV-Con) or Klotho-

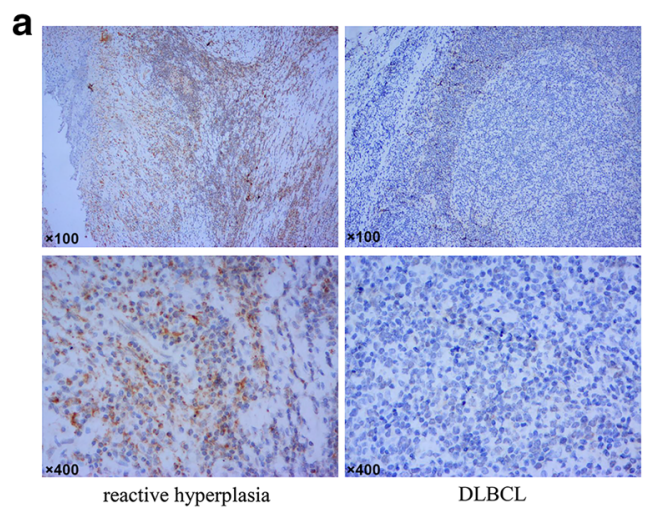

C

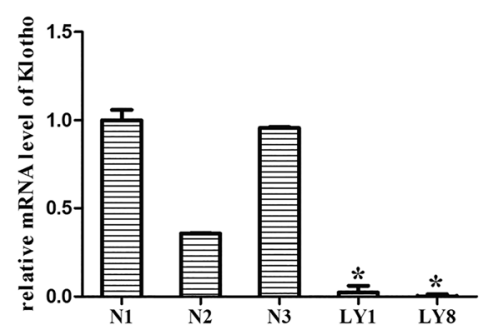

b

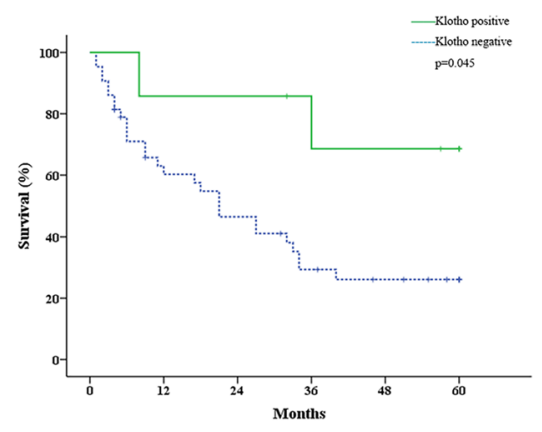

d

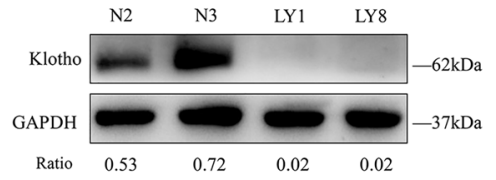

Fig. 1 Klotho was downregulated in DLBCL and related to tumor progression. a Compared with reactive hyperplasia, expression level of Klotho was significantly decreased in DLBCL tissues. Original magnification, $\times 100$ (upper panel) and $\times 400$ (lower panel). b Patients with Klotho negative expression showed shorter survival than those with Klotho positive expression. c As detected by real-time quantitative PCR, lower levels of Klotho mRNA expression were observed in DLBCL cell lines (LY1, LY8) than in CD19+ B cells (N1, N2, N3) (mean $\left.\pm S D, n=3,{ }^{*} p<0.05\right)$. d Protein expression levels of Klotho were detected in DLBCL cells and normal PBMCs. The ratios of relative protein expression level of targets are indicated below the western blot 
Table 1 Correlation between Klotho expression and clinical characteristics of DLBCL patients

\begin{tabular}{|c|c|c|c|c|}
\hline \multirow[t]{2}{*}{ Characteristics } & \multirow[t]{2}{*}{ No. of patients } & \multicolumn{2}{|c|}{ Klotho expression } & \multirow[t]{2}{*}{$p$ value } \\
\hline & & Positive (\%) & Negative & \\
\hline \multicolumn{5}{|l|}{ Age (years) } \\
\hline$<60$ & 27 & $4(14.8 \%)$ & 23 & \multirow[t]{2}{*}{0.857} \\
\hline$\geq 60$ & 23 & $3(13.0 \%)$ & 20 & \\
\hline \multicolumn{5}{|l|}{ Gender } \\
\hline Male & 26 & $2(7.7 \%)$ & 24 & \multirow[t]{2}{*}{0.181} \\
\hline Female & 24 & $5(20.8 \%)$ & 19 & \\
\hline \multicolumn{5}{|c|}{ Ann Arbor stage } \\
\hline | or || & 12 & $5(41.7 \%)$ & 7 & \multirow[t]{2}{*}{0.002} \\
\hline III or IV & 38 & $2(5.3 \%)$ & 36 & \\
\hline \multicolumn{5}{|l|}{ B symptoms } \\
\hline Present & 14 & $2(14.3 \%)$ & 12 & \multirow[t]{2}{*}{0.971} \\
\hline Absent & 36 & $5(13.9 \%)$ & 31 & \\
\hline \multicolumn{5}{|l|}{ Subtype } \\
\hline GCB & 15 & $2(13.3 \%)$ & 13 & \multirow[t]{2}{*}{0.929} \\
\hline Non-GCB & 35 & $5(14.3 \%)$ & 30 & \\
\hline \multicolumn{5}{|l|}{ Serum LDH } \\
\hline Normal & 35 & $5(14.3 \%)$ & 30 & \multirow[t]{2}{*}{0.929} \\
\hline Elevated & 15 & $2(13.3 \%)$ & 13 & \\
\hline \multicolumn{5}{|c|}{ Extranodal involvement } \\
\hline Absent & 28 & $4(14.3 \%)$ & 18 & \multirow[t]{2}{*}{0.45} \\
\hline Present & 22 & $3(13.7 \%)$ & 25 & \\
\hline \multicolumn{5}{|l|}{ IPI score } \\
\hline $0-2$ & 35 & $6(17.1 \%)$ & 29 & \multirow[t]{2}{*}{0.328} \\
\hline $3-5$ & 15 & $1(6.7 \%)$ & 14 & \\
\hline
\end{tabular}

$L D H$ lactate dehydrogenase, IPI international prognostic index

overexpression lentivirus vectors (LV-KL). The upregulation of Klotho levels were confirmed by quantitative PCR and western blot (Fig. 2a, b). Cell proliferation rates were evaluated by CCK-8 assay. Significant reduction of cell proliferation was observed in LY1 and LY8 cells transfected with LV-KL, compared with those transfected with empty vector (Fig. 2c). Furthermore, we investigated the anti-tumor effect of Klotho on DLBCL xenografts in vivo. LY1 cells, stably expressing Klotho, or the vector control cells were injected subcutaneously into SCID Beige mice, respectively. Consistent with the in vitro results, mice treated with Klotho overexpression cells revealed remarkable reduction in tumor volume compared to that transfected with empty vector (Fig. 2d). Higher expression level of Klotho was identified in mice treated with LV-KL-transfected LY1 cells (Fig. 2e). In addition, we estimated the expression level of proliferative marker Ki67 $[27,28]$ in xenograft mice by IHC. Lower level of Ki67 positive rate were observed in LV-KL group (Fig. 2e).

\section{Klotho promoted apoptosis of DLBCL cells}

To investigate whether Klotho could promote the apoptosis of DLBCL cells, Annexin-V-based apoptotic assays were performed. Flow cytometry analysis indicated that Klotho overexpression resulted in enforced apoptosis rates in both LY1 $(6.56 \pm 0.71 \%$ in LV-Con vs. $16.41 \pm 2.16 \%$ in LV-KL group, $p=0.002$ ) and LY8 cells $(7.23 \pm 0.65 \%$ in $\mathrm{LV}$-Con vs. $16.03 \pm 1.85 \%$ in $\mathrm{LV}-\mathrm{KL}$ group, $p=0.001$, Fig. 3a, b). In addition, western blot analysis confirmed the pro-apoptotic effects of Klotho. Dramatically reduction of anti-apoptotic protein Mcl-1 and increased cleaved forms of Caspase-3 were observed in DLBCL cell lines (Fig. 3c). Altogether, these results indicate that upregulation of Klotho could promote the apoptosis of DLBCL cells.

\section{Klotho modulated the activation of IGF-1R signaling in DLBCL}

Having shown that Klotho could impair cell proliferation and induce apoptosis in DLBCL, we next investigated the molecular mechanisms responsible for the function of Klotho. The IGF-1R pathway plays a vital role in the development of hematological malignancies [14, 25, 29]. CCK-8 assay was conducted to assess the effect of Klotho on IGF-1-induced cell proliferation. DLBCL cells transfected with either LV-KL or LV-Con were treated with IGF-1 or vehicle control in $0.5 \%$ FBS culture medium for 24-96 h. In the groups untreated with IGF-1, LV-KL transfection resulted in declined proliferation of LY1 and LY8 cells compared to emptyvector group. In the IGF-1-treated groups, we observed that cell proliferation was less restored by IGF-1 in cells transfected with LV-KL compared to that transfected with LV-Con. Following addition of IGF-1, cell proliferation of LV-Con-treated cells increased by up to $60 \%$, whereas the only up to $40 \%$ enhancement of cell proliferation was found in LV-KL transfected cells (Fig. 4a).

As the IGF-1R signaling could be activated by IGF-1, we explored the optimal activation dose and time of IGF-1 in DLBCL cell lines. LY1 cells were transfected with either LV-KL or LV-Con, serum starved for $48 \mathrm{~h}$ and treated with IGF-1 for the indicated times and doses. Western blot was carried out to evaluate the phosphorylation level of the IGF-1R. As shown in Fig. 4b, the maximum activation occurred at IGF-1 concentration of $50 \mathrm{ng} / \mathrm{ml}$ and at the time of $30 \mathrm{~min}$ after treatment. Then, we studied the ability of Klotho to modulate activation of IGF-1R signaling in DLBCL cells. Cells transfected with either LV-KL or LV-Con were serum starved for $48 \mathrm{~h}$, treated with IGF-1 $(50 \mathrm{ng} / \mathrm{ml}$ for $30 \mathrm{~min})$ or vehicle control. Following treatment, cells were harvested and immunoblotting was conducted. Decreased phosphorylation 


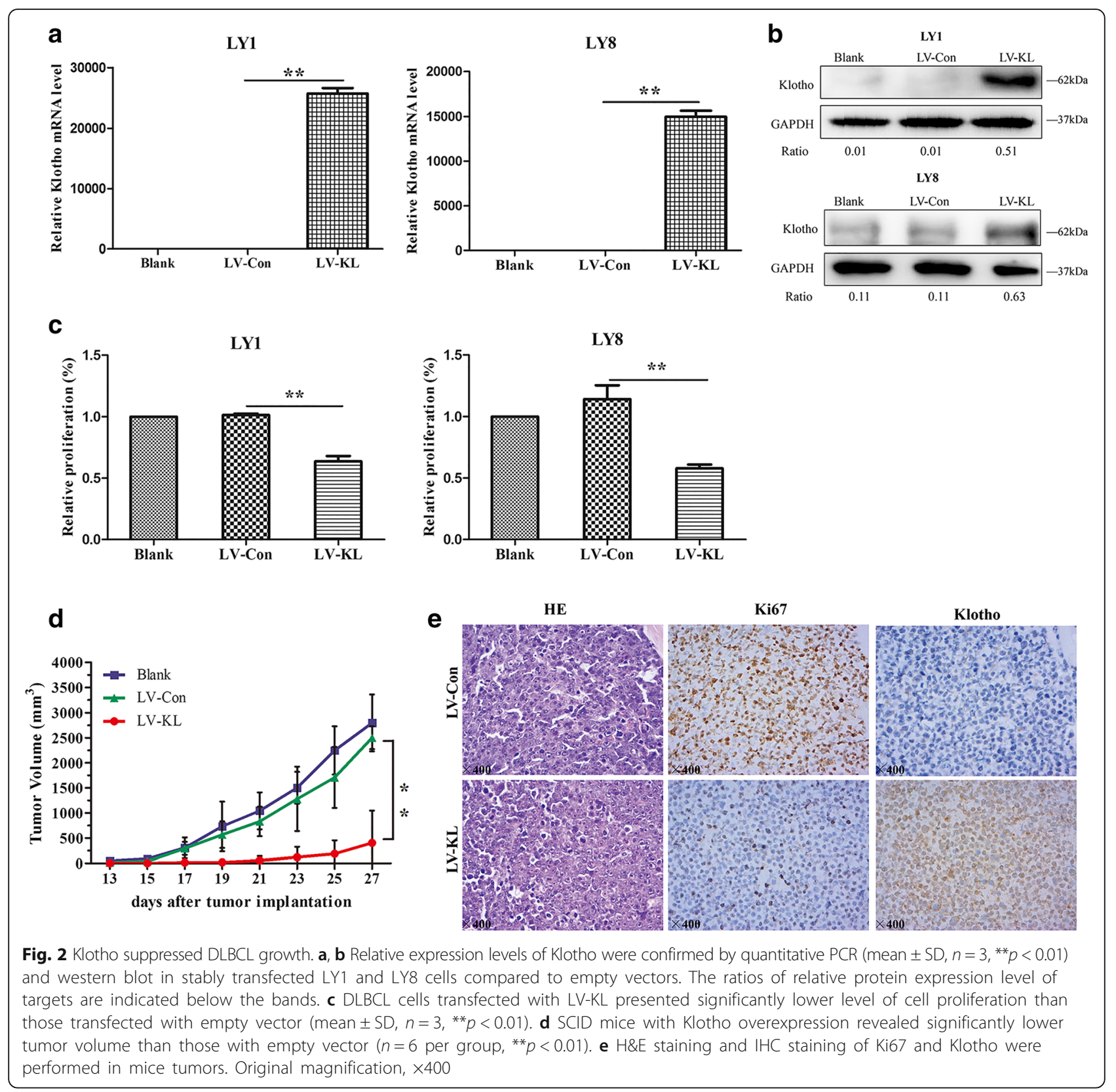

level of IGF-1R and its downstream targets, including AKT and ERK1/2, were observed in cells transfected with LV-KL (Fig. 4c). Furthermore, we evaluated the modulation of Klotho on IGF-1R signaling in DLBCL xenograft model. Enhanced expression of Klotho in LV-KL-treated mice was confirmed by immunoblotting (Fig. 5d). Decreased phosphorylation of IGF1-R as well as its downstream targets were observed in mice treated with LV-KL compared to the control group (Fig. 4d). These results demonstrated that Klotho may act as a modulator of IGF-1R signaling contributed to the tumorigenesis of DLBCL (Fig. 4e).
rhKL acted as an active form both in vitro and in vivo

We further investigated the activity of secreted Klotho protein on DLBCL. To explore the effect of secreted Klotho on proliferation of DLBCL cells, LY1 and LY8 cells were seeded and treated with rhKL. The rhKL was found to be active and reduced the proliferative ability of DLBCL cells by $30 \sim 40 \%$ at the concentration of $2 \mu \mathrm{g} / \mathrm{ml}$ (Fig. 5a, b). ADR is one of the most commonly used drugs in the chemotherapeutic strategy of DLBCL. We detected the function of Klotho on cell responses to ADR. LY1 and LY8 cells were treated for $48 \mathrm{~h}$ with rhKL, ADR, or their combination, and cell proliferation was then evaluated. 


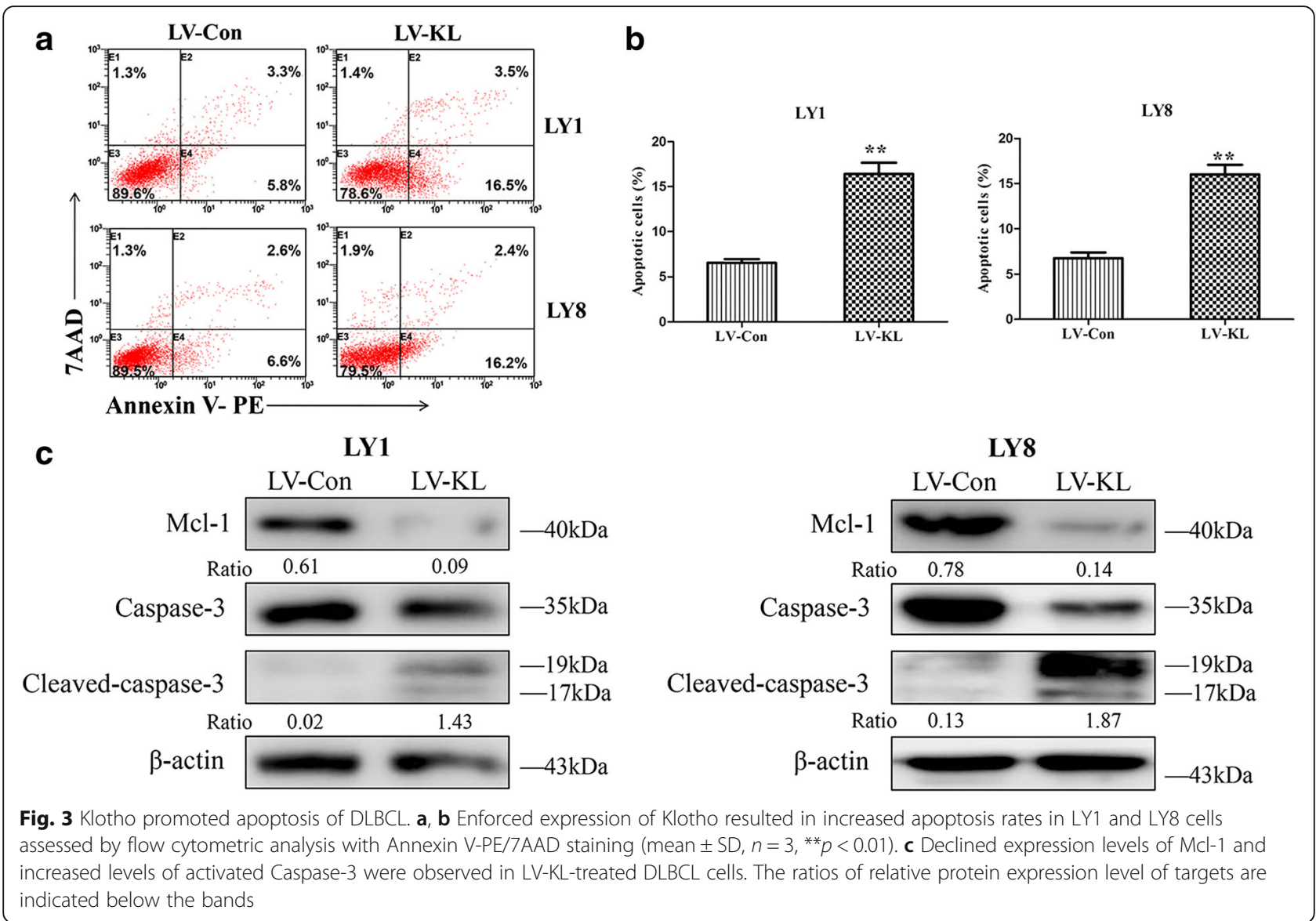

Combination of rhKL $(2 \mu \mathrm{g} / \mathrm{ml})$ increased the sensitivity of DLBCL cells to ADR (Fig. 5a, b).

The in vivo activity of rhKL in DLBCL xenograft model was also detected. LY1 cells were injected subcutaneously into the SCID Beige mice to build a DLBCL xenograft model. After 15 days, the mice were treated with daily intraperitoneal injection of either rhKL $(7.5 \mu \mathrm{g} / \mathrm{kg}, n=6)$ or a control vehicle $(n=6)$ for 2 weeks. Significant decreased tumor volumes were observed in the mice treated with rhKL compared with those treated with vehicle control (Fig. 5c). Moreover, reductive expression level of proliferative marker Ki67 was found in rhKL-treated mice (Fig. 5d).

In addition, we employed ELISA assays to test the soluble Klotho levels in the serum of 30 initially diagnosed DLBCL patients and 15 healthy volunteers. Lower serum Klotho levels were noted in DLBCL patients $(628.54 \pm 219.39 \mathrm{pg} / \mathrm{ml})$ than the control subjects $(818.87 \pm 241.51 \mathrm{pg} / \mathrm{ml}, p=0.045$, Fig. $5 \mathrm{e})$.

\section{Discussion}

In this study, our observations identified for the first time that Klotho, an anti-aging gene, as a potential tumor suppressor in DLBCL tumorigenesis. Klotho was downregulated in human DLBCL and inhibited the growth of DLBCL both in vitro and in vivo. Upregulation of Klotho resulted in declined activation of IGF-1R signaling pathway in DLBCL.

We identified remarkable reduced expression of Klotho in DLBCL tissues and cell lines, whereas higher expression in reactive hyperplasia and $\mathrm{CD} 19+\mathrm{B}$ cells from normal donors. Decreased level of Klotho was associated with advanced stage and more aggressive disease process in DLBCL. Tumor suppressive activity of Klotho has been reported in several human solid malignancies, but never in hematological cancers [30-33]. Recent investigations elucidated that epigenetic mechanisms, including promoter methylation and histone deacetylation, contributed to the reduced Klotho expression in human breast cancer, cervical cancer, and hepatocellular carcinomas [34-36]. The similar mechanism may be involved in DLBCL. At present, the diagnosis of DLBCL is mainly based on the biopsy histopathology and IHC [37]. With the deepening of additional studies, lower expression of Klotho may serve as a potential marker for the pathological diagnosis of DLBCL.

DLBCL is a high-grade heterogeneous disorder defined by heterogeneity in clinical and biological characteristics 


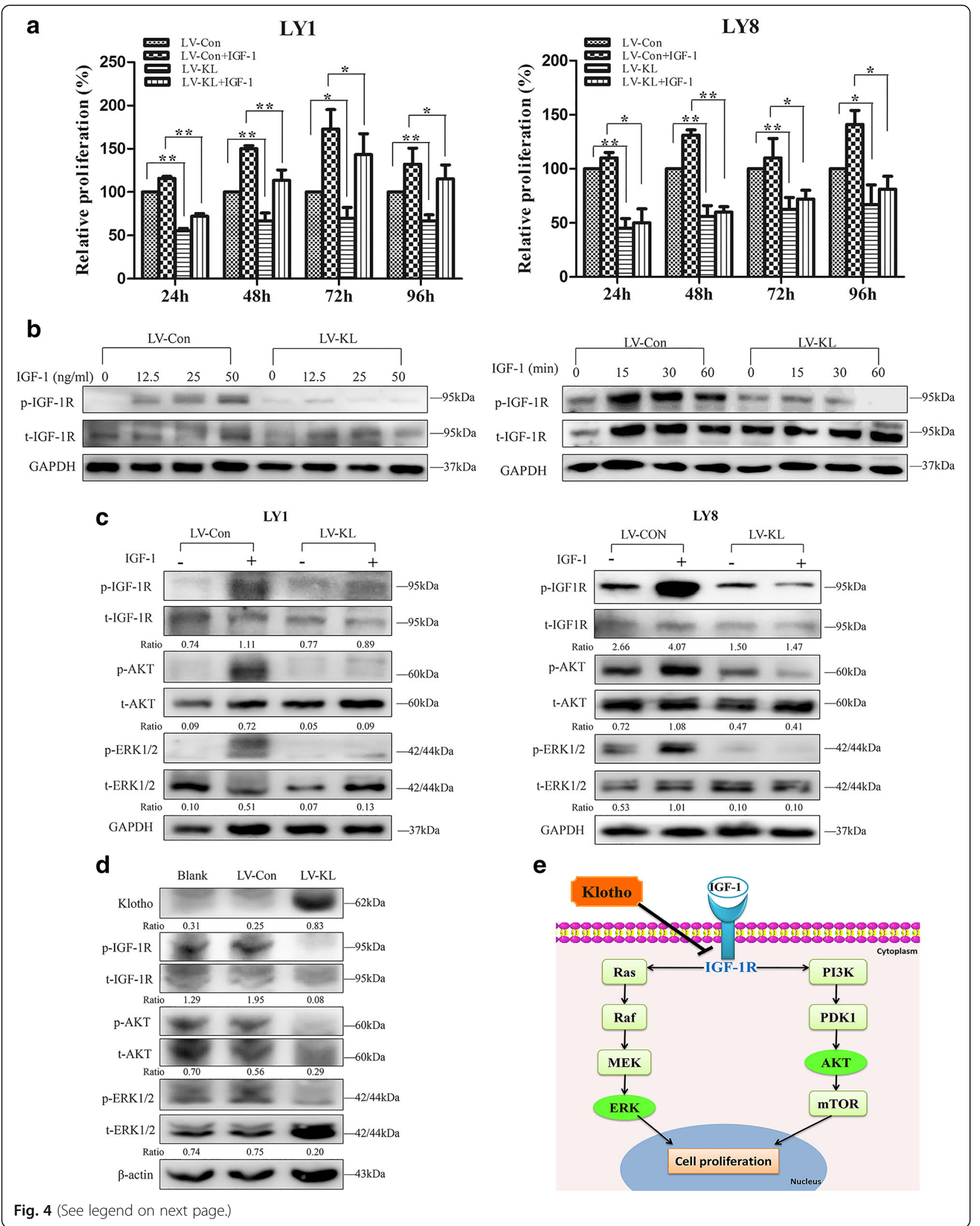


(See figure on previous page.)

Fig. 4 Klotho modulated activation of IGF-1R pathway in DLBCL. a IGF-1-induced DLBCL cell (LY1 and LY8) proliferation is inhibited by Klotho overexpression. LY1 and LY8 cells were transfected with LV-KL or LV-Con, starved for $48 \mathrm{~h}$ and treated by IGF-1 (50 ng/ml) and analyzed by CCK-8 assay (mean $\pm \mathrm{SD}, n=3,{ }^{*} p<0.05,{ }^{* *} p<0.01$ ). $\mathbf{b} \mathrm{LY} 1$ with stable transfection of LV-KL or LV-Con were serum starved for $48 \mathrm{~h}$ and treated with IGF-1 $(50 \mathrm{ng} / \mathrm{ml})$ for the indicated times or IGF-1 $(30 \mathrm{~min})$ for the indicated doses. After treatment, cells were harvested and analyzed by western blot. c LY1 and LY8 cells transfected with LV-KL or LV-Con, serum starved for $48 \mathrm{~h}$, and treated with IGF-1 (50 ng/ml, 30 min). Western blot was conducted to assess the phosphorylated ( $p$ ) and total (t) protein levels of IGF-1R, AKT, and ERK1/2. The ratios of relative protein expression level of targets are indicated below the bands. $\mathbf{d}$ Decreased activation of IGF1-R signaling was observed in LV-KL-treated mice. The ratios of relative protein expression level of targets are indicated below the bands. e Schematic description of Klotho mediated IGF1R signaling

$[1,38,39]$. Personalized prognostic stratification and targeted therapeutic strategies are urgently needed to improve the outcomes of DLBCL patient [40]. Ki67 as a proliferative marker performed as a poor prognostic marker in DLBCL [27]. In this study, we discovered that DLBCL xenograft mice with Klotho overexpression exhibited significantly lower Ki67 staining positive rate than that without Klotho upregulation.

As Klotho exists in both membrane-bound form and secreted form, the secreted Klotho could be shed and released into the circulation [7]. Recent investigation elucidated the low serum level of Klotho in renal cell

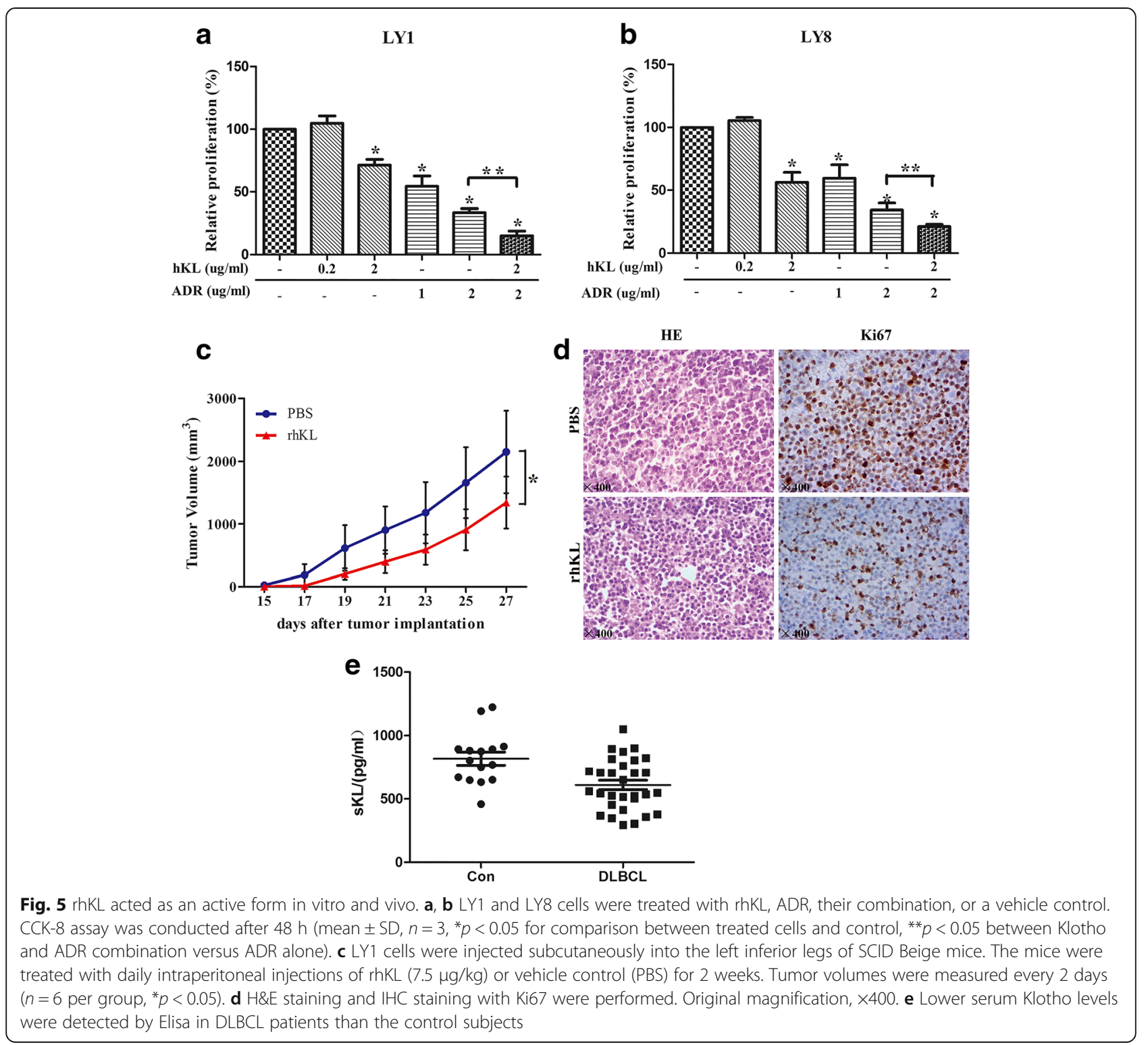


carcinoma [41]. Low serum Klotho level was an independent adverse prognostic factor for cancer-specific and progression-free survival in RCC [41]. However, the level of serum soluble Klotho was unchanged in multiple myeloma [42]. Thus, the diagnostic role of secreted Klotho in human cancer remains controversial. In this study, we identified the decreased level of serum Klotho in DLBCL patients. The correlation of serum Klotho with disease diagnosis and progression still needs further exploration. Larger number of the included patients will better confirm the role of serum Klotho in DLBCL.

Importantly, our study illuminated that Klotho effectively inhibited the growth of DLBCL cells. Overexpression of Klotho significantly inhibited cell proliferation and induced cell apoptosis in DLBCL. We also discovered that efficacy of ADR could be enhanced by combination with rhKL in DLBCL cells. This finding suggested the potential of Klotho in therapeutic intervention of DLBCL. Our in vivo investigations demonstrated that upregulation of Klotho, either by LV-KL transfection or rhKL administration, congruously led to inhibitory effect in the tumor growth of xenograft model of DLBCL. This will pave the way for rational design of Klotho-based molecular products in DLBCL. Nevertheless, pharmacokinetic investigations are still required to explore the optimal dose and adverse reaction of rhKL in the use of either single agent treatment or drug combination.

Significantly, we also found that Klotho could inhibit the activation of IGF-1R signaling in DLBCL. Induction of IGF-1R signaling was involved in the pathogenesis of hematological malignancies [13]. Recently, Stromberg et al. reported the involvement of IGF-1R and the antitumor effects of specific IGF-1R inhibitors in DLBCL tumorigenesis [25]. Structure-function analysis of Klotho indicated that Klotho could interact with the IGF-1R [43]. Klotho-induced inhibition of IGF-1R signaling may act as a novel mechanism involved in the development of DLBCL. Apart from IGF-1R signaling, many other pathways have been confirmed to be modulated by Klotho in tumorigenesis. The most studied is the FGFRKlotho axis, and Klotho could act as cofactor of endocrine FGFs to bind and induce the activation of FGFRs in breast, pancreatic, and prostate cancers [11, 30, 44]. Deregulation of Wnt signaling pathway plays a critical role in the pathogenesis of cancers [45-47]. It was proved that Klotho participated in tumorigenesis partly through restraining the Wnt signaling pathway [48]. The modulation of Klotho on these pathways in DLBCL is poorly understood. In spite of the important discoveries revealed by these studies, there are also limitations on the detailed mechanisms and the cross-talks of them involved in Klotho deregulation.

\section{Conclusions}

Taken together, our findings identified that Klotho performs as tumor suppressor and modulator of IGF-1R signaling in the DLBCL. Overexpression of Klotho may be a predictive marker for favorable outcome in DLBCL. Klotho reinforces the response of DLBCL cells to chemotherapeutic drug. Being an endogenous circulating hormone, the secreted Klotho could function as an active form and inhibit the tumor growth effectively both in vitro and in vivo. This study illuminates Klotho as a potential target for future therapeutic strategies.

\section{Abbreviations \\ ADR: Adriamycin; CCK-8: Cell Counting Kit-8; DAB: Diaminobenzidine; DLBCL: Diffuse large B cell lymphoma; FGF23: Fibroblast growth factor 23; GFP: Green fluorescent protein; H\&E: Hematoxylin-eosin; IGF-1R: Insulin-like growth factor-1 receptor; IHC: Immunohistochemistry; IPI: International prognostic index; LDH: Lactate dehydrogenase; LV-Con: Negative control lentivirus vectors; LV-KL: Klotho-overexpression lentivirus vectors; NHL: Non-Hodgkin lymphoma; PBMCs: Peripheral blood mononuclear cells; PBS: Phosphate buffer solution; PFA: Paraformaldehyde; rhKL: Recombinant human Klotho; RTKs: Receptor tyrosine kinases; SABC: Strept avidin-horseradish peroxidase complex; SCID: Severe combined immunodeficiency}

\section{Acknowledgements}

Not applicable.

\section{Funding}

This study was supported by National Natural Science Foundation (No. 81473486 and No. 81270598), National Public Health Grand Research Foundation (No. 201202017), Natural Science Foundations of Shandong Province (No. ZR2012HZ003 and No. 2009ZRB14176), Technology Development Projects of Shandong Province (No. 2014GSF118021, No. 2010GSF10250, and No. 2008GG2NS02018), Program of Shandong Medical Leading Talent, and Taishan Scholar Foundation of Shandong Province.

\section{Availability of data and materials}

All data generated and analyzed during this study are included in this published article.

\section{Authors' contributions}

$X X Z$ and $X W$ conceived and designed the experiments. XXZ, XSF, YJJ, and $L Y G$ performed the experiments. $X Y L, Y L, K L, P P L$, and $X L$ assisted with the performance of experiments. $X X Z, X S F$ and $Y J J$ analyzed the data. XXZ and XW wrote the paper. All authors read and approved the final manuscript.

\section{Competing interests}

The authors declare that they have no competing interests.

\section{Consent for publication}

Not applicable.

\section{Ethics approval and consent to participate}

This study was approved by the Medical Ethical Committee of Shandong Provincial Hospital affiliated to Shandong University. All samples of patients were obtained with informed consent in accordance with the Declaration of Helsinki. All animal procedures described in this article have been approved by the Animal Care and Use Committee of Shandong Provincial Hospital affiliated to Shandong University.

Received: 18 October 2016 Accepted: 3 January 2017

Published online: 02 February 2017

\section{References}

1. Roschewski M, Staudt LM, Wilson WH. Diffuse large B-cell lymphoma-treatment approaches in the molecular era. Nat Rev Clin Oncol. 2014;11:12-23. 
2. Zhang J, Grubor V, Love CL, Banerjee A, Richards KL, Mieczkowski PA, et al. Genetic heterogeneity of diffuse large B-cell lymphoma. Proc Natl Acad Sc U S A. 2013;110:1398-403.

3. Horn H, Ziepert M, Wartenberg M, Staiger AM, Barth TF, Bernd HW, et al Different biological risk factors in young poor-prognosis and elderly patients with diffuse large B-cell lymphoma. Leukemia. 2015;29:1564-70.

4. Saito B, Shiozawa E, Usui T, Nakashima H, Maeda T, Hattori N, et al. Rituximab with chemotherapy improves survival of non-germinal center type untreated diffuse large B-cell lymphoma. Leukemia. 2007;21:2563-6.

5. Sehn LH, Gascoyne RD. Diffuse large B-cell lymphoma: optimizing outcome in the context of clinical and biologic heterogeneity. Blood. 2015;125:22-32.

6. Kuro-o M, Matsumura Y, Aizawa H, Kawaguchi H, Suga T, Utsugi T, et al. Mutation of the mouse klotho gene leads to a syndrome resembling ageing. Nature. 1997;390:45-51.

7. Kurosu H, Yamamoto M, Clark JD, Pastor JV, Nandi A, Gurnani P, et al. Suppression of aging in mice by the hormone Klotho. Science. 2005;309:1829-33.

8. Kuro-o M. Klotho. Pflugers Arch. 2010;459:333-43.

9. Kuro-o M. Klotho and aging. Biochim Biophys Acta. 2009;1790:1049-58.

10. Yamamoto M, Clark JD, Pastor JV, Gurnani P, Nandi A, Kurosu H, et al. Regulation of oxidative stress by the anti-aging hormone klotho. J Biol Chem. 2005:280:38029-34.

11. Wolf I, Levanon-Cohen S, Bose S, Ligumsky H, Sredni B, Kanety $H$, et al. Klotho: a tumor suppressor and a modulator of the IGF-1 and FGF pathways in human breast cancer. Oncogene. 2008;27:7094-105.

12. Zhou X, Wang X. Klotho: a novel biomarker for cancer. J Cancer Res Clin Oncol. 2015;141:961-9.

13. Yaktapour N, Ubelhart R, Schuler J, Aumann K, Dierks C, Burger M, et al. Insulin-like growth factor-1 receptor (IGF1R) as a novel target in chronic lymphocytic leukemia. Blood. 2013;122:1621-33.

14. Vishwamitra D, Shi P, Wilson D, Manshouri R, Vega F, Schlette EJ, et al. Expression and effects of inhibition of type I insulin-like growth factor receptor tyrosine kinase in mantle cell lymphoma. Haematologica. 2011;96:871-80.

15. Mathur R, Sehgal L, Braun FK, Berkova Z, Romaguerra J, Wang M, et al. Targeting Wnt pathway in mantle cell lymphoma-initiating cells. J Hematol Oncol. 2015;8:63.

16. Lam BQ, Dai L, Qin Z. The role of HGF/C-MET signaling pathway in lymphoma. J Hematol Oncol. 2016;9:135.

17. Toffalini F, Demoulin JB. New insights into the mechanisms of hematopoietic cell transformation by activated receptor tyrosine kinases. Blood. 2010;116:2429-37.

18. Xie J, Chen X, Zheng J, Li C, Stacy S, Holzenberger M, et al. IGF-IR determines the fates of BCR/ABL leukemia. J Hematol Oncol. 2015;8:3.

19. Pollak MN, Schernhammer ES, Hankinson SE. Insulin-like growth factors and neoplasia. Nat Rev Cancer. 2004;4:505-18.

20. Dong S, Qu X, Li W, Zhong X, Li P, Yang S, et al. The long non-coding RNA, GAS5, enhances gefitinib-induced cell death in innate EGFR tyrosine kinase inhibitor-resistant lung adenocarcinoma cells with wide-type EGFR via downregulation of the IGF-1R expression. J Hematol Oncol. 2015;8:43.

21. Herrero-Sanchez MC, Rodriguez-Serrano C, Almeida J, San Segundo L, Inoges S, Santos-Briz A, et al. Targeting of PI3K/AKT/mTOR pathway to inhibit T cell activation and prevent graft-versus-host disease development. J Hematol Oncol. 2016:9:113.

22. Lonetti A, Cappellini A, Bertaina A, Locatelli F, Pession A, Buontempo F, et al. Improving nelarabine efficacy in $T$ cell acute lymphoblastic leukemia by targeting aberrant PI3K/AKT/mTOR signaling pathway, J Hematol Oncol. 2016;9:114.

23. Roberts PJ, Der CJ. Targeting the Raf-MEK-ERK mitogen-activated protein kinase cascade for the treatment of cancer. Oncogene. 2007;26:3291-310.

24. Vishwamitra D, George SK, Shi P, Kaseb AO, Amin HM. Type I insulin-like growth factor receptor signaling in hematological malignancies. Oncotarget. 2016; doi: 10.18632/oncotarget.12123.

25. Stromberg T, Feng $X$, Delforoush M, Berglund $M$, Lin $Y$, Axelson $M$, et al. Picropodophyllin inhibits proliferation and survival of diffuse large B-cell lymphoma cells. Med Oncol. 2015;32:188.

26. Jaffe ES. The 2008 WHO classification of lymphomas: implications for clinical practice and translational research. Hematology Am Soc Hematol Educ Program. 2009:523-531. doi: 10.1182/asheducation-2009.1.523.

27. Szczuraszek K, Mazur G, Jelen M, Dziegiel P, Surowiak P, Zabel M. Prognostic significance of Ki-67 antigen expression in non-Hodgkin's lymphomas. Anticancer Res. 2008;28:1113-8.
28. Scholzen T, Gerdes J. The Ki-67 protein: from the known and the unknown. J Cell Physiol. 2000;182:311-22

29. Whelan JT, Ludwig DL, Bertrand FE. HoxA9 induces insulin-like growth factor-1 receptor expression in B-lineage acute lymphoblastic leukemia. Leukemia. 2008;22:1161-9.

30. Abramovitz L, Rubinek T, Ligumsky H, Bose S, Barshack I, Avivi C, et al. KL1 internal repeat mediates klotho tumor suppressor activities and inhibits bFGF and IGF-I signaling in pancreatic cancer. Clin Cancer Res. 2011;17:4254-66.

31. Chen B, Ma X, Liu S, Zhao W, Wu J. Inhibition of lung cancer cells growth, motility and induction of apoptosis by Klotho, a novel secreted Wnt antagonist, in a dose-dependent manner. Cancer Biol Ther. 2012;13:1221-8.

32. Li XX, Huang LY, Peng JJ, Liang L, Shi DB, Zheng HT, et al. Klotho suppresses growth and invasion of colon cancer cells through inhibition of IGF1R-mediated PI3K/AKT pathway. Int J Oncol. 2014;45:611-8.

33. Aviel-Ronen S, Rubinek T, Zadok O, Vituri A, Avivi C, Wolf I, et al. Klotho expression in cervical cancer: differential expression in adenocarcinoma and squamous cell carcinoma. J Clin Pathol. 2016;69:53-7.

34. Rubinek T, Shulman M, Israeli S, Bose S, Avraham A, Zundelevich A, et al. Epigenetic silencing of the tumor suppressor klotho in human breast cancer. Breast Cancer Res Treat. 2012;133:649-57.

35. Xie B, Zhou J, Yuan L, Ren F, Liu DC, Li Q, et al. Epigenetic silencing of Klotho expression correlates with poor prognosis of human hepatocellular carcinoma. Hum Pathol. 2013:44:795-801.

36. Lee J, Jeong DJ, Kim J, Lee S, Park JH, Chang B, et al. The anti-aging gene $\mathrm{KLOTHO}$ is a novel target for epigenetic silencing in human cervical carcinoma. Mol Cancer. 2010:9:109.

37. Tilly H, Gomes da Silva M, Vitolo U, Jack A, Meignan M, Lopez-Guillermo A, et al. Diffuse large B-cell lymphoma (DLBCL): ESMO Clinical Practice Guidelines for diagnosis, treatment and follow-up. Ann Oncol. 2015;26 Suppl 5:v116-25.

38. Intlekofer AM, Younes A. Precision therapy for lymphoma-current state and future directions. Nat Rev Clin Oncol. 2014;11:585-96.

39. Martelli M, Ferreri AJ, Agostinelli C, Di Rocco A, Pfreundschuh M, Pileri SA. Diffuse large B-cell lymphoma. Crit Rev Oncol Hematol. 2013;87:146-71.

40. Vermaat JS, Pals ST, Younes A, Dreyling M, Federico M, Aurer I, et al. Precision medicine in diffuse large B-cell lymphoma: hitting the target. Haematologica. 2015;100:989-93.

41. Gigante M, Lucarelli G, Divella C, Netti GS, Pontrelli P, Cafiero C, et al. Soluble serum alphaKlotho is a potential predictive marker of disease progression in clear cell renal cell carcinoma. Medicine (Baltimore). 2015;94:e1917.

42. Suvannasankha A, Tompkins DR, Edwards DF, Petyaykina KV, Crean CD, Fournier PG, et al. FGF23 is elevated in multiple myeloma and increases heparanase expression by tumor cells. Oncotarget. 2015;6:19647-60.

43. Ligumsky H, Rubinek T, Merenbakh-Lamin K, Yeheskel A, Sertchook R, Shahmoon S, et al. Tumor suppressor activity of Klotho in breast cancer is revealed by structure-function analysis. Mol Cancer Res. 2015;13:1398-407.

44. Feng S, Dakhova O, Creighton CJ, Ittmann M. Endocrine fibroblast growth factor FGF19 promotes prostate cancer progression. Cancer Res. 2013;73:2551-62.

45. McCubrey JA, Steelman LS, Bertrand FE, Davis NM, Abrams SL Montalto G, et al. Multifaceted roles of GSK-3 and Wnt/beta-catenin in hematopoiesis and leukemogenesis: opportunities for therapeutic intervention. Leukemia. 2014;28:15-33.

46. Ugarte GD, Vargas MF, Medina MA, Leon P, Necunir D, Elorza AA, et al. Wnt signaling induces transcription, spatial proximity, and translocation of fusion gene partners in human hematopoietic cells. Blood. 2015;126:1785-9.

47. Wu C, Zhang HF, Gupta N, Alshareef A, Wang Q, Huang YH, et al. A positive feedback loop involving the Wnt/beta-catenin/MYC/Sox2 axis defines a highly tumorigenic cell subpopulation in ALK-positive anaplastic large cell lymphoma. J Hematol Oncol. 2016;9:120.

48. Sun H, Gao Y, Lu K, Zhao G, Li X, Li Z, et al. Overexpression of Klotho suppresses liver cancer progression and induces cell apoptosis by negatively regulating wnt/ beta-catenin signaling pathway. World J Surg Oncol. 2015;13:307. 